\title{
Diffusion of Brownian particles governed by fluctuating friction
}

\author{
J. Łuczka ${ }^{\mathrm{a}}$, P. Talkner ${ }^{\mathrm{b}, *}$, P. Hänggi ${ }^{\mathrm{c}}$ \\ ${ }^{a}$ Institute of Physics, University of Silesia, 40-007 Katowice, Poland \\ ${ }^{\mathrm{b}}$ General Energy Research, Paul Scherrer Institut, CH-5232 Villigen, Switzerland \\ ${ }^{\mathrm{c} I n s t i t u t e}$ of Physics, Universität Augsburg, Universitätsstr. 1, D-86135 Augsburg, Germany
}

Received 23 September 1999

\begin{abstract}
The problem of diffusion of Brownian particles is revisited under the condition of fluctuactions of the Stokes friction, which depends on the viscosity of the medium surrounding the Brownian particle, as well as on its shape and size. Various classes of discontinuous and continuous stochastic processes describing fluctuating friction are considered. All these cases lead to normal diffusion. The diffusion constant, however, deviates from the usual Einstein form: in particular it generically depends on the Brownian particle's mass. For all considered cases it is a decreasing function of mass. (c) 2000 Elsevier Science B.V. All rights reserved.
\end{abstract}

PACS: 02.50.Ey; 05.40.-a; 05.40.Jc; 05.60.cd; 46.65+g

Keywords: Brownian motion; Fluctuating friction; Mass-dependent diffusion

\section{Introduction}

Diffusion plays an important role in a variety of physical transport mechanisms $[1,2]$. A hallmark of diffusion theory is the Einstein relation which connects the diffusion constant to the friction coefficient [3]. In this case it turns out that the diffusion constant actually becomes independent of the mass $m$ of the Brownian particle: it solely depends on its geometry and the viscosity and temperature of the environment. This conventional description of diffusion, however, breaks down in dense fluids if the particle density and the density of the surrounding medium become of comparable order. A typical example is the dynamics of a Brownian particle being described by the Basset-Boussinesq

\footnotetext{
${ }^{*}$ Corresponding author. Fax: +41-56-310-4412.

E-mail address: talkner@psi.ch (P. Talkner)
} 
equation (which is valid when the density of the fluid is of the same order as of the particle). The corresponding diffusion constant then reads [4]

$$
D=D_{E}\left(1+2 \frac{m_{f}}{m}\right)
$$

where $D_{E}$ is the (mass-independent) Einstein diffusion constant and $m_{f}$ denotes the mass of the fluid displaced by the diffusing particle. Yet another example is provided by the diffusing aggregate of fractal dimension $d_{f}$ for which $D \propto m^{-d_{f}}$ [5]. This relation has been derived theoretically [6], obtained from simulations [7] and confirmed experimentally [8].

In this work we find another situation where the diffusion constant becomes generically dependent on the diffusing particle's own mass. The situation is provided by a thermal equilibrium dynamics which satisfies detailed balance symmetry with a fluctuating friction coefficient [9]. For example, this can be realized with particles diffusing in ferroelectric fluids when external stochastic magnetic fields are controling the intrinsic viscosity. Generically, the viscosity can be manipulated in an a priori manner in systems possessing a fluctuating resistance value. Some specific examples that come to mind are the giant magnetic resistance phenomenon [10], or, likewise, the recently discovered colossal magnetic resistance effect [11]. Another class is provided by solid-state systems wherein the damping is governed by an internal resistance.

It has been shown previously [9] that the effective diffusion in fluctuating media is bounded from below by the conventional Einstein result $D_{E}$ and from above by its average:

$$
D_{E}=k_{B} T /\langle\gamma\rangle \leqslant D \leqslant\langle D\rangle=k_{B} T\left\langle\gamma^{-1}\right\rangle,
$$

wherein $\gamma$ denotes the fluctuating friction coefficient and the brackets indicate the average over the corresponding ensemble. In the following, we generalize the investigation of Ref. [9] for dichotomic fluctuations to more general discrete fluctuations of shot noise and Kubo-Anderson type as well as to a positive valued continuous process. In doing so we derive a general relationship between the dynamical properties of the friction fluctuations and the effective (position) diffusion constant. In Section 2 we review the model dynamics of diffusion driven by fluctuating friction. Section 3 contains the material providing the above mentioned connection between the friction dynamics and diffusion constant. In the remaining section we present explicit results for various discrete and continuous stochastic friction dynamics.

\section{Set up of model dynamics}

We consider here the motion of a Brownian particle of mass $m$ which experiences a fluctuating friction $\gamma(z)$. Following the reasoning in Ref. [9] we investigate the 
following extended Langevin equation:

$$
\begin{aligned}
& \dot{x}=v, \\
& m \dot{v}=-\gamma(z) v+\sqrt{2 \gamma(z) k_{B} T} f(t),
\end{aligned}
$$

which satisfies via the fluctuation-dissipation relation the detailed balance symmetry. In (2.1) a dot denotes a derivative with respect to time $t$, and $f(t)$ is a Gaussian white noise source, satisfying,

$$
\langle f(t)\rangle=0, \quad\langle f(t) f(s)\rangle=\delta(t-s) .
$$

We assume that $z=z(t)$ is an environmental variable which influences the friction coefficient but does neither depend on the state $(x, v)$ of the Brownian particle nor on the fluctuating force $f(t)$. In particular, this means that we neglect any backreaction of the state of the particle on the environment that would influence the viscosity. We note that the friction coefficient must be non-negative for all realizations of the environmental variable $z(t)$. For the sake of simplicity only, we assume a stationary and Markovian time evolution of the environmental variable $z(t)$, with the fluctuating friction given by

$$
\gamma(z)=\gamma_{0}+z(t) \geqslant 0 .
$$

Thus, $z(t)$ is bounded from below by $-\gamma_{0}$. Its mean value $\langle z(t)\rangle$ does not depend on time and its correlation function $\langle z(t) z(s)\rangle=\langle z(t-s) z(0)\rangle$ only depends on the time-difference $(t-s)$.

We focus here on the behavior of the particle's mean square displacement $\left\langle x^{2}(t)\right\rangle$ in the limit of long times $t$. In this context the behavior of the long-time limit of the position-velocity correlation function $\lim _{t \rightarrow \infty}\langle x(t) v(t)\rangle$ is important. It can be found from the formal solution of the Langevin equation (2.1), yielding

$$
v(t)=\mathrm{e}^{-\Gamma(t)} v_{0}+\int_{0}^{t} \mathrm{~d} s \mathrm{e}^{-(\Gamma(t)-\Gamma(s))} \sqrt{\frac{2 \dot{\Gamma}(s) k_{B} T}{m}} f(s) .
$$

Here $v_{0}$ is the initial velocity and $\Gamma(t)$ denotes the integrated friction, i.e.,

$$
\Gamma(t)=\frac{1}{m} \int_{0}^{t} \mathrm{~d} s \gamma(z(s)) .
$$

A further integration yields the position $x(t)$. For the position-velocity correlation function one then obtains after some algebra:

$$
\begin{aligned}
\langle x(t) v(t)\rangle= & \left\langle x_{0} v_{0}\right\rangle\left\langle\mathrm{e}^{-\Gamma(t)}\right\rangle+\left(\left\langle v_{0}^{2}\right\rangle-\frac{k_{B} T}{m}\right) \int_{0}^{t} \mathrm{~d} s\left\langle\mathrm{e}^{-\Gamma(t)-\Gamma(s)}\right\rangle \\
& +\frac{k_{B} T}{m} \int_{0}^{t} \mathrm{~d} s\left\langle\mathrm{e}^{-\Gamma(s)}\right\rangle,
\end{aligned}
$$

where $x_{0}$ denotes the initial position.

From the three terms of the right-hand side the third one dominates the long-time behavior of the velocity-position correlation function (for details see Appendix A). It is 
therefore our first goal to express the mean-value $\langle\exp \{-\Gamma(s)\}\rangle$ and its time-integral in terms of the viscosity fluctuations $z(t)$. For this purpose consider the integrated process,

$$
Z(t)=\int_{0}^{t} \mathrm{~d} s z(s)
$$

and its corresponding probability density $\rho(Z, t)$. Once we know $\rho(Z, t)$, the mean value of $\langle\exp \{-\Gamma(s)\}\rangle$ follows from the characteristic function of $Z(t)$ :

$$
\tilde{\rho}(k, t)=\left\langle\mathrm{e}^{\mathrm{i} k Z(t)}\right\rangle=\int \mathrm{d} Z \mathrm{e}^{\mathrm{i} k Z} \rho(Z, t),
$$

as

$$
\left\langle\mathrm{e}^{-\Gamma(t)}\right\rangle=\mathrm{e}^{-\gamma_{0} t / m} \tilde{\rho}(i / m, t) .
$$

The long-time behavior can often be inferred from the Laplace transform of the characteristic function:

$$
\hat{\rho}(k, u)=\int_{0}^{\infty} \mathrm{d} t \mathrm{e}^{-u t} \tilde{\rho}(k, t) .
$$

In the case of ordinary diffusion the velocity position correlation approaches the diffusion constant in the limit $t \rightarrow \infty$,

$$
D=\lim _{t \rightarrow \infty} \frac{1}{2} \frac{d\left\langle x^{2}(t)\right\rangle}{d t}=\lim _{t \rightarrow \infty}\langle x(t) v(t)\rangle .
$$

and consequently from (2.6) we find as a first main result

$$
D=\frac{k_{B} T}{m} \int_{0}^{\infty} \mathrm{d} t \mathrm{e}^{-\gamma_{0} t / m}\left\langle\mathrm{e}^{-Z(t) / m}\right\rangle=\frac{k_{B} T}{m} \hat{\rho}\left(i / m, \gamma_{0} / m\right) .
$$

Although we will not meet an example of anomalous diffusion in the sequel, for the sake of completeness, we give here also the expressions of the diffusion constant for such cases. Anomalous diffusion is charcterized by an algebraic growth of the variance of the position being slower than linear in time for subdiffusion and faster for superdiffusion [12], i.e.,

$$
\left\langle x^{2}(t)\right\rangle=2 D_{\alpha} t^{\alpha} .
$$

For the anomalous diffusion constant one then finds the relation

$$
D_{\alpha}=\frac{1}{\alpha \Gamma(\alpha)} \lim _{u \rightarrow 0^{+}} u^{\alpha-1} \hat{\rho}\left(i / m, u+\gamma_{0} / m\right) .
$$

For $\alpha=1$, Eq. (2.12) is readily recovered.

\section{Relationship between friction dynamics and the characteristic function}

As a stationary Markovian process, the conditional probability of the viscosity fluctuations fulfills a master equation (forward equation)

$$
\frac{\partial}{\partial t} p\left(z, t \mid z^{\prime}\right)=\Lambda p\left(z, t \mid z^{\prime}\right),
$$


where $\Lambda$ is the master operator of the considered process acting on the $z$-dependence of the conditional probability $p\left(z, t \mid z^{\prime}\right)$. The combined process $\{Z(t), z(t)\}$ is also Markovian. In view of the fact that

$$
\dot{Z}(t)=z(t), \quad Z(0)=0,
$$

the joint probability $p(Z, z ; t)$ of the pair $\{Z(t), z(t)\}$ obeys the extended master equation:

$$
\frac{\partial}{\partial t} p(Z, z ; t)=-\frac{\partial}{\partial Z} z p(Z, z ; t)+\Lambda p(Z, z ; t)
$$

with the initial condition

$$
p(Z, z ; 0)=\delta(Z) p_{\mathrm{st}}(z),
$$

where $\delta(x)$ is the Dirac $\delta$-function and $p_{\mathrm{st}}(z)$ the stationary distribution of the viscosity fluctuations, obeying

$$
\Lambda p_{\mathrm{st}}(z)=0 \text {. }
$$

The initial distribution of the process $Z(t)$ follows from (3.2). The marginal probability $\rho(Z, t)$ follows from $p(Z, z ; t)$ by an integration over $z$ :

$$
\rho(Z, t)=\int_{\Omega} \mathrm{d} z p(Z, z ; t)
$$

where $\Omega$ is the phase-space of the fluctuations $z(t)$. Upon a Fourier transformation with respect to $Z$ and a Laplace transform with respect to time $t$ one obtains for

$$
\hat{p}(k, z ; u)=\int \mathrm{d} Z \int_{0}^{\infty} \mathrm{d} t \mathrm{e}^{\mathrm{i} k Z-u t} p(Z, z ; t),
$$

the following inhomogeneous equation:

$$
(u-\mathrm{i} k z-\Lambda) \hat{p}(k, z ; u)=p_{\mathrm{st}}(z) .
$$

For normal diffusion the diffusion constant in (2.12) emerges as

$$
\begin{aligned}
D & =\frac{k_{B} T}{m} \int_{\Omega} \hat{p}\left(\mathrm{i} / m, z ; \gamma_{0} / m\right) \mathrm{d} z \\
& =k_{B} T \int_{\Omega} \frac{1}{\gamma_{0}+z-m \Lambda} p_{\mathrm{st}}(z) \mathrm{d} z,
\end{aligned}
$$

where Eq. (3.6) has been used. In the second line we have inserted the formal solution of Eq. (3.8). This relation establishes the general connection between the diffusion constant $D$ and the stationary stochastic friction dynamics. In the next section we consider selected examples of models for fluctuations $z(t)$ for which the solution of Eq. (3.8) can be obtained, and an explicit form of the diffusion constant $D$ can be derived. 


\section{Examples}

\subsection{Delta-correlated shot noise}

In this subsection we consider white shot noise as a model of the friction fluctuations $z(t)$. Because the characteristic function of integrated white shot noise is known, the diffusion constant (2.12) can immediately be determined. White shot noise is defined as [13]

$$
z(t)=\sum_{i=1}^{n(t)} a_{i} \delta\left(t-t_{i}\right)-\lambda\langle a\rangle,
$$

where the random instants $t_{i}$ are the arrival times of a Poissonian counting process $n(t)$ with parameter $\lambda$; i.e., the probability that $k \delta$-impulses occur within time $t$ is given by the Poissonian distribution $\operatorname{Pr}\{n(t)=k\}=(\lambda t)^{k} \exp (-\lambda t) / k$ !. Accordingly, the duration $s$ between successive Poissonian arrival times, $s=t_{i}-t_{i-1}$, is exponentially distributed with the probability density $\lambda \exp (-\lambda s)$. The parameter $\lambda$ determines the mean number of the $\delta$-impulses per unit time; it equals the reciprocal average sojourn time $\tau_{\text {so }}=1 / \lambda$ between two $\delta$-impulses. The positive-valued amplitudes $\left\{a_{i}\right\}$ of the $\delta$-impulses are identically distributed random variables with mean-value $\langle a\rangle$. They are independent of each other, and of the counting process $n(t)$. The common probability density of the weights $\left\{a_{i}\right\}$ is denoted by $w(a), a>0$. The sum of weighted $\delta$-impulses on the right-hand side of Eq. (4.1) is compensated on average by the bias

$$
z_{0}=\lambda\langle a\rangle \text {. }
$$

In order that the friction constant (2.3) is non-negative we have $z_{0} \leqslant \gamma_{0}$.

From definition (4.1) it follows that the shot noise has a vanishing mean value and is $\delta$-correlated; i.e.,

$$
\langle z(t)\rangle=0, \quad\langle z(t) z(u)\rangle=\lambda\left\langle a^{2}\right\rangle \delta(t-u),
$$

where $\left\langle a^{2}\right\rangle$ denotes the variance of the amplitudes $a_{i}$. The characteristic function $\tilde{\rho}(k, t)$ of the integral of process (4.1) can be expressed in terms of the characteristic function $\tilde{w}(t)$ of the amplitudes of the shot noise

$$
\tilde{w}(k)=\int_{0}^{\infty} \mathrm{d} a \mathrm{e}^{\mathrm{i} k a} w(a) .
$$

It reads [14]

$$
\tilde{\rho}(k, t)=\exp \left[-\mathrm{i} k z_{0} t\right] \exp \{\lambda[\tilde{w}(k)-1] t\} .
$$

The Laplace transform of the characteristic function with respect to $t$ is easily obtained. With (2.12) the diffusion constant yields the result

$$
D=\frac{k_{B} T}{m}\left\{\frac{\gamma_{0}-\lambda\langle a\rangle}{m}+\lambda[1-\tilde{w}(\mathrm{i} / m)]\right\}^{-1},
$$


which still depends on the yet unspecified distribution $w(a)$ of the $\delta$-impulse amplitudes. The assumption of positive friction, i.e., $\gamma_{0}>z_{0}=\lambda\langle a\rangle$ (cf. (2.3)) and the relation $1-\tilde{w}(\mathrm{i} / m) \geqslant 0$ ensure a positive value of $D$ in (4.6).

As an example, we assume a Gamma distribution of the amplitudes; i.e.,

$$
w(a)=\frac{1}{B^{\mu} \Gamma(\mu)} a^{\mu-1} \mathrm{e}^{-a / B} \Theta(a),
$$

where the exponent $\mu>0$, the scale parameter $B>0, \Gamma(x)$ stands for the Gamma function, and $\Theta(x)$ denotes the Heaviside step function, respectively. The moments of $a$ become $\left\langle a^{r}\right\rangle=B^{r} \Gamma(\mu+r) / \Gamma(\mu)$. The most probable value is found at $a=0$ for $0<\mu \leqslant 1$ and at $a=(\mu-1) B$ for $\mu \geqslant 1$. Eq. (4.6) then takes the form

$$
D=D_{E} \gamma_{0}\left\{\gamma_{0}-\mu \lambda B+m \lambda\left[1-\left(\frac{m}{m+B}\right)^{\mu}\right]\right\}^{-1},
$$

where

$$
D_{E}=\frac{k_{B} T}{\langle\gamma(z)\rangle}
$$

is Einstein's diffusion constant of ordinary Brownian motion with the average friction coefficient given by $\langle\gamma(z)\rangle=\gamma_{0}$.

In the particular case of $\mu=1$ one obtains an exponential distribution of amplitudes with a most probable value located at zero, $\langle a\rangle=B$ and the bias $z_{0}=\lambda B<\gamma_{0}$. The diffusion constant (4.8) then reduces to the simple expression

$$
D=D_{E}\left[1+\frac{\lambda B}{\gamma_{0}-\lambda B+m \gamma_{0} / B}\right] .
$$

In contrast to Einstein's diffusion constant $D_{E}, D$ depends on mass! The diffusion constant possesses the same mass dependence as for dichotomic noise [9]. With increasing mass the diffusion constant approaches $D_{E}$ from above, being in accordance with the generally valid inequality $D \geqslant D_{E}$ (cf. (1.2)).

For $\mu=2$ the corresponding diffusion constant results as

$$
D=D_{E}\left[1+\frac{2 \lambda B^{3}+3 m \lambda B^{2}}{\left(\gamma_{0}-2 \lambda B\right)(m+B)^{2}+m \lambda B(2 m+B)}\right] .
$$

This case depicts a more complex dependence on mass $m$.

\subsection{Kubo-Anderson fluctuations}

The Kubo-Anderson process is a particular case of the large class of kangaroo stochastic processes. It is a purely discontinuous (Kolmogorov-Feller) stationary stochastic process for which the transition probability per unit time $P\left(z_{2} \mid z_{1}\right)$ for a jump from the state $z_{1}$ into the state $z_{2}$ is independent of the initial state [15], i.e.,

$$
P\left(z_{2} \mid z_{1}\right)=q\left(z_{2}\right) v_{0} .
$$

Here, we have introduced the normalized probability density $q(z)$ of final states and the jump-rate $v_{0}$ which by definition is independent of the initial state. The waiting-time 
distribution is exponential and thus the mean waiting time reads $\tau_{w}=1 / v_{0}$ for all states. Accordingly, the master-operator $\Lambda$ governing this process acts on the probability density $p(z, t)$ of the process $z(t)$ as

$$
\Lambda p(z, t)=-v_{0} p(z, t)+v_{0} q(z) \int_{\Omega} p(y, t) \mathrm{d} y .
$$

Note that $\Lambda q(z)=0$, and consequently $q(z)$ is the stationary probability density of the process $z(t)$

$$
p_{\mathrm{st}}(z)=q(z) \text {. }
$$

In order to determine the diffusion constant, we have to solve Eq. (3.8) which now takes the form

$$
\left(z+m v_{0}+\gamma_{0}\right) \hat{p}\left(\mathrm{i} / m, z ; \gamma_{0} / m\right)-m v_{0} q(z) \int_{\Omega} \hat{p}\left(\mathrm{i} / m, y, \gamma_{0} / m\right) \mathrm{d} y=m q(z),
$$

where we have used the relation (4.14). This is an integral equation with respect to $\hat{p}\left(\mathrm{i} / m, z, \gamma_{0} / m\right)$ with a degenerate kernel [16]. It can readily be solved to give

$$
\hat{p}\left(\mathrm{i} / m, z, \gamma_{0}\right)=\frac{m}{1-m v_{0} A} \frac{q(z)}{z+m v_{0}+\gamma_{0}},
$$

where

$$
A=\int_{\Omega} \frac{q(z) \mathrm{d} z}{z+m v_{0}+\gamma_{0}} .
$$

With (3.9) one obtains for the diffusion constant the expression:

$$
D=k_{B} T \frac{A}{1-m v_{0} A} .
$$

The diffusion constant depends via the constant $A$ on the still unspecified jumpdistribution $q(z)$ for which we consider two examples:

(1) For a uniform jump distribution on the interval $\Omega=\left[-z_{0}, z_{0}\right], 0<z_{0}<\gamma_{0}$ the average friction becomes $\langle\gamma(z)\rangle=\gamma_{0}$, and the constant $A$ is given by

$$
A=\frac{1}{2 z_{0}} \ln \frac{m v_{0}+\gamma_{0}+z_{0}}{m v_{0}+\gamma_{0}-z_{0}} .
$$

(2) As an example of a jump-distribution that is unbounded from above we consider the Gamma distribution defined on $\Omega=[0, \infty)$

$$
q(z)=p_{\text {st }}(z)=\frac{1}{c^{\mu} \Gamma(\mu)} z^{\mu-1} \mathrm{e}^{-z / c} \Theta(z),
$$

where the exponent $\mu$ and the scale-parameter $c$ are positive. In this case the averaged friction coefficient is

$$
\langle\gamma(z)\rangle=\gamma_{0}+\mu c
$$

and

$$
A=c^{-1}\left(\tilde{m}+\tilde{\gamma}_{0}\right)^{\mu-1} \mathrm{e}^{\tilde{m}+\tilde{\gamma_{0}}} \Gamma\left(1-\mu, \tilde{m}+\tilde{\gamma}_{0}\right),
$$

where $\tilde{m}=m v_{0} / c$ and $\tilde{\gamma}_{0}=\gamma_{0} / c$ denote rescaled dimensionless mass and reference friction, respectively, and $\Gamma(a, x)$ stands for the incomplete Gamma function. 


\subsection{Continuous fluctuations}

Here, we present a model of continuous friction fluctuations. Let us define the process $z(t)$ by the Ito stochastic differential equation [17]

$$
\dot{z}=\omega-\beta z+\sqrt{2 \varepsilon z} f_{1}(t), \quad z \in \Omega=[0, \infty),
$$

where the parameters $\omega, \beta$ and $\varepsilon$ are positive and $f_{1}(t)$ is delta-correlated Gaussian noise of zero mean and with the same correlations as in (2.2). This process results from the transformation $z=y^{2}$ of the amplitude part of a Rayleigh process $y(t)$ which is defined by [18]

$$
\dot{y}(t)=-\frac{\beta}{2} y(t)+\frac{\omega-2 \varepsilon}{2 y(t)}+\sqrt{2 \varepsilon} f_{1}(t) .
$$

For the particular case $\omega=\varepsilon / 2, y(t)$ becomes an Ornstein-Uhlenbeck process. In Appendix B the spectral representation of the Fokker-Planck operator and the transition probability are presented. Moreover it is shown that the process $z(t)$ is exponentially correlated. Its stationary probability distribution $p_{\mathrm{st}}(z)$ is given by a Gamma distribution (4.20) with the parameters

$$
\mu=\omega / \varepsilon, \quad c=\varepsilon / \beta .
$$

Hence, the averaged friction coefficient is given by (4.21). In order to find the solution of Eq. (3.8) we proceed in two steps. First, we split off the stationary distribution from the Laplace transformed characteristic function $\hat{p}\left(\mathrm{i} / m, z ; \gamma_{0} / m\right)$

$$
\hat{p}\left(\mathrm{i} / m, z ; \gamma_{0} / m\right)=p_{\text {st }}(z) W(z)
$$

and obtain for the remaining function $W(z)$

$$
\left[\varepsilon z \frac{\mathrm{d}^{2}}{\mathrm{~d} z^{2}}+\omega \frac{\mathrm{d}}{\mathrm{d} z}-\beta z \frac{\mathrm{d}}{\mathrm{d} z}-\gamma_{0} / m-z / m\right] W(z)=-1 .
$$

This procedure results in a non-homogeneous ordinary differential equation of second order. In a second step we solve (4.27) by setting for $W(z)$ the ansatz

$$
W(z)=\int_{h_{0}}^{h_{1}} \mathrm{e}^{-z s} \phi(s) \mathrm{d} s,
$$

where $\phi(s)$ is an unknown function and the interval of integration needs to be specified. Upon insertion into Eq. (4.27) we find the relation

$$
\begin{aligned}
\int_{h_{0}}^{h_{1}} & \mathrm{e}^{-z s}\left\{\left(\varepsilon s^{2}+\beta s-1 / m\right) \frac{\mathrm{d} \phi(s)}{\mathrm{d} s}+\left[(2 \varepsilon-\omega) s+\beta-\gamma_{0} / m\right] \phi(s)\right\} \mathrm{d} s \\
- & \left.\mathrm{e}^{-z s}\left[\varepsilon s^{2}+\beta s-1 / m\right] \phi(s)\right|_{h_{0}} ^{h_{1}}=-1 .
\end{aligned}
$$

This equation holds if two conditions are satisfied: the expression in the curly bracket under the integral vanishes and the last (boundary) term is equal to -1 . The former condition yields a first-order differential equation for the function $\phi(s)$, namely,

$$
\left(\varepsilon s^{2}+\beta s-1 / m\right) \frac{\mathrm{d} \phi(s)}{\mathrm{d} s}=\left[(\omega-2 \varepsilon) s-\beta+\gamma_{0} / m\right] \phi(s) .
$$


Table 1

Dimensionless parameters for fluctuationg friction models; $\mu$ and $\tilde{m}$ may only take positive values; the allowed ranges of $\tilde{\gamma}_{0}$ are indicated

\begin{tabular}{llll}
\hline & Shot noise & Kubo-Anderson & Continuous noise \\
\hline$\mu$ & $\mu$ & $\mu$ & $\omega / \varepsilon$ \\
$\tilde{\gamma_{0}}$ & $\gamma_{0} /(\mu \lambda B) \in[1, \infty)$ & $\gamma_{0} / c \in[0, \infty)$ & $\gamma_{0} \beta / \varepsilon \in[0, \infty)$ \\
$\tilde{m}$ & $m / B$ & $m v_{0} / c$ & $m \beta^{2} / \varepsilon$ \\
\hline
\end{tabular}

Its solution reads

$$
\phi(s)=\phi(0)\left|1-\frac{s}{s_{1}}\right|^{\delta}\left|1-\frac{s}{s_{2}}\right|^{\mu-\delta-2},
$$

where

$$
\begin{aligned}
& s_{1 / 2}= \pm \frac{c}{2}(\Delta \mp 1), \quad \Delta=(1+4 c / m \beta)^{1 / 2}, \\
& \delta=\mu / 2-1+\left(\gamma_{0} / m \beta-\mu / 2\right) / \Delta .
\end{aligned}
$$

The second condition is fulfilled if one assumes that $h_{0}=0, \phi(0)=m$ (which gives an initial condition for the differential equation (4.30)) and $h_{1}$ is one of two roots $\left\{s_{1}, s_{2}\right\}$. We choose the positive root $s_{1}$ because $W(z)$ must be positive.

The diffusion constant $D$ is obtained from $W(z)$ via the expression

$$
D=\frac{k_{B} T}{m \Gamma(\mu)} \int_{0}^{\infty} \mathrm{e}^{-z} z^{\mu-1} W(c z) \mathrm{d} z .
$$

Inserting (4.28) into this equation and integrating over the $z$-variable yields the result:

$$
D=\frac{k_{B} T}{c} \int_{0}^{s_{0}}\left(1-\frac{s}{s_{0}}\right)^{\delta}\left(1+\frac{s}{1+s_{0}}\right)^{\mu-\delta-2}(1+s)^{-\mu} \mathrm{d} s,
$$

where $s_{0}=s_{1} c=(\Delta-1) / 2$. The integral exists for all values of parameters because $\delta>-1$.

\section{Diffusion with fluctuating friction: discussion of example cases}

For all presented models of friction fluctuations, the diffusion constant $D$ is a monotonically decreasing function of mass $m$ which approaches the Einstein diffusion constant (4.9) as $m$ grows to infinity. All our examples are characterized by three dimensionless parameters: an exponent $\mu$, a dimensionless reference friction $\tilde{\gamma}_{0}$ and a dimensionless mass $\tilde{m}$ (see Table 1). For the delta-correlated shot noise fluctuations with Gamma distributed amplitudes, the diffusion can be rescaled to read

$$
\frac{D}{D_{E}}=\tilde{\gamma}_{0}\left[\tilde{\gamma}_{0}-1+\frac{\tilde{m}}{\mu}\left[1-\left(\frac{\tilde{m}}{1+\tilde{m}}\right)^{\mu}\right]\right]^{-1} .
$$




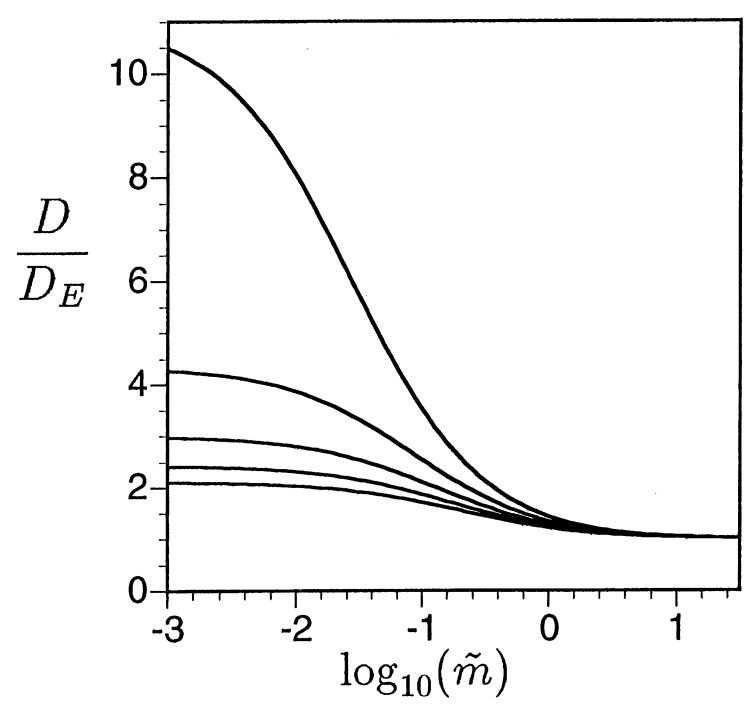

Fig. 1. $D / D_{E}$ for white shot-noise with Gamma distributed impulse strengths as a function $\operatorname{of} \log _{10}(\tilde{m})$ for different values of $\tilde{\gamma}_{0}=1.1,1.3,1.5,1.7,1.9$ (from top to bottom) and $\mu=0.1$.

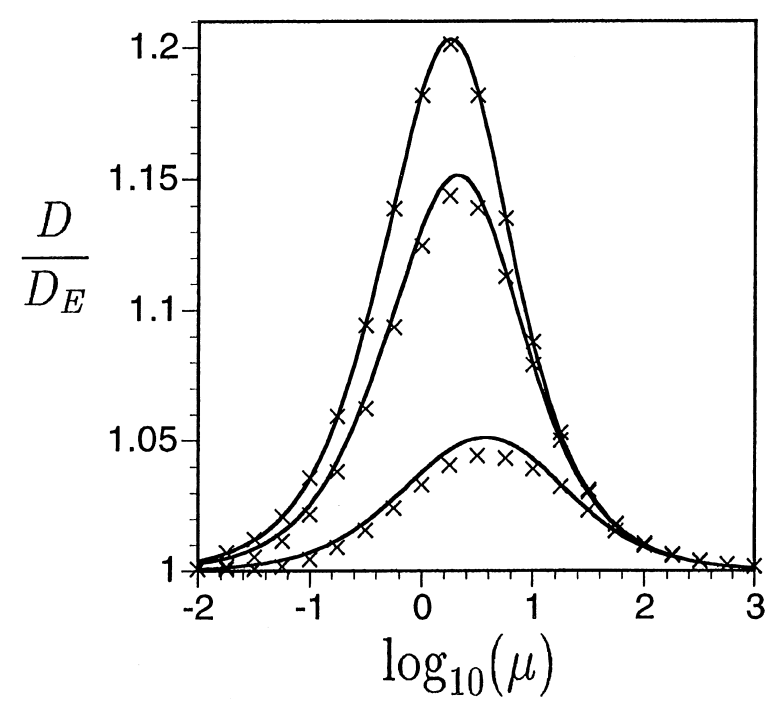

Fig. 2. $D / D_{E}$ for Kubo-Anderson friction driven by Gamma distributed noise (solid lines) and for continuous friction fluctuations (crosses) as a function of $\log _{10}(\mu)$ for $\tilde{\gamma}_{0}=1$ and $\tilde{m}=0.1,1,10$ (from top to bottom).

Fig. 1 depicts the behavior of $D / D_{E}$ versus the dimensionless mass $\tilde{m}$ for five different values of the reference friction $\tilde{\gamma}_{0}$. The diffusion constant (5.1) is a smooth function of all parameters which approaches finite values in all limiting cases. It monotonically decreases as a function of both particle mass $\tilde{m} \in(0, \infty)$, and reference friction $\tilde{\gamma}_{0} \in[1, \infty)$. 


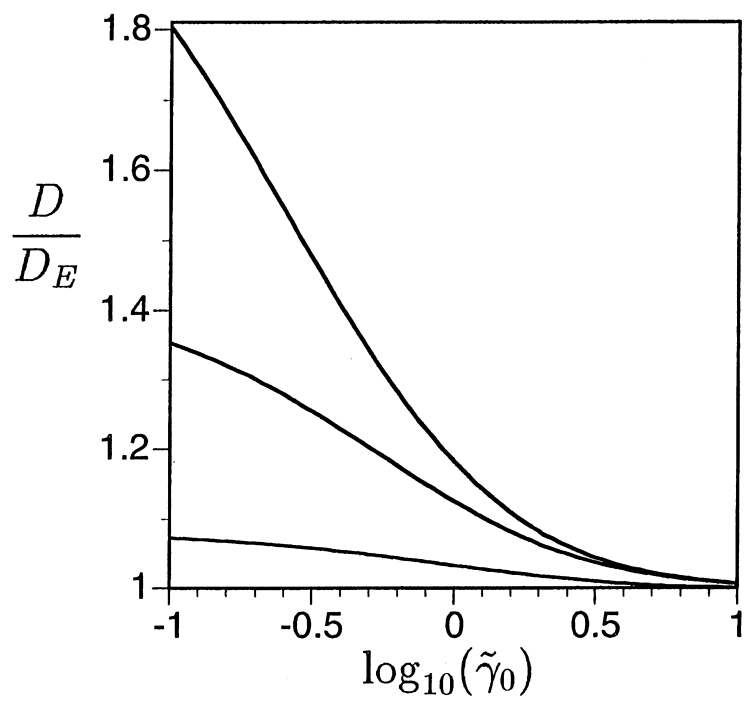

Fig. 3. $D / D_{E}$ for continuous friction fluctuations as a function of $\log _{10}\left(\tilde{\gamma}_{0}\right)$ for $\mu=1$ and $\tilde{m}=0.1,1,10$ (from top to bottom).

The cases with discontinuous friction fluctuations of the Kubo-Anderson type and positive-valued continuous fluctuations of the type of Eq. (4.23) are depicted with Figs. 2 and 3. From Fig. 2 we deduce that the diffusion ratio $D / D_{E}$ is always enhanced in accordance with general inequality (1.2) but can be controlled selectively as a function of the exponent $\mu$ of the Gamma distribution. There is only a minor difference between the diffusion enhancements of these two fluctuating friction models which coincide in the distribution of friction values but differ with respect to their dynamics. The enhancement reaches maximal values for a small Brownian particle mass $\tilde{m} \rightarrow 0$ with $D / D_{E}=\tilde{\gamma}_{0}^{\mu-1}\left(\tilde{\gamma}_{0}+\mu\right) \mathrm{e}^{\tilde{\gamma}_{0}} \Gamma\left(1-\mu, \tilde{\gamma}_{0}\right)$ for Kubo-Anderson friction fluctuations. The monotonically decreasing dependence on the reference friction $\tilde{\gamma}_{0}$ is shown in Fig. 3 for continuous friction fluctuations. The maximal enhancement of $D / D_{E}$ is achieved for small reference friction $\gamma_{0}$ and small mass $\tilde{m}$.

\section{Acknowledgements}

J.Ł. acknowledges support by the Deutsche Physikalische Gesellschaft (Bavarian Regional Section) and the Komitet Badań Naukowych, Poland (Grant No. 2P03B 160 17), and P. H. the support by the German Israeli Foundation (G.I.F. No. G-411-018.05/95).

\section{Appendix A. Long-time behavior of the velocity-position correlation}

We assume that the stationary process $\gamma(t)$ is ergodic and that its mean value $\langle\gamma\rangle$ is positive. Because of the ergodicity, the process $\gamma(t)$ takes almost all of its 
possible values within sufficiently long intervals. Consequently, the integrated process $\Gamma(t)$ defined in Eq. (2.5) grows in time without bound and $\mathrm{e}^{-\Gamma(t)}$ monotonically decreases and approaches zero in the limit $t \rightarrow \infty$. The $t \rightarrow \infty$-limit of the position-velocity correlation function (2.6) consequently becomes:

$$
\lim _{t \rightarrow \infty}\langle x(t) v(t)\rangle=\frac{k_{B} T}{m} \int_{0}^{\infty}\left\langle\mathrm{e}^{-\Gamma(s)}\right\rangle \mathrm{d} s .
$$

\section{Appendix B. Multiplicative model of continuous viscosity fluctuations}

The stochastic process $z(t)$ defined by Eq. (4.23) is a stationary Markovian diffusion process. Its time-evolution is governed by the Fokker-Planck operator

$$
\Lambda=-\frac{\partial}{\partial z}(\omega-\beta z)+\varepsilon \frac{\partial^{2}}{\partial z^{2}} z
$$

Its normalized stationary solution is given by (4.20). The transition probability $p\left(z, t \mid z_{0}, s\right) \equiv p\left(z, t-s \mid z_{0}\right)$ can be expressed in terms of the eigenvalues $-n \beta, n=$ $0,1,2, \ldots$, left eigenfunctions $L_{n}^{\mu-1}(z / c)$ and right eigenfunctions $n ! \Gamma(\mu) /$ $\Gamma(\mu+n) L_{n}^{\mu-1}(z / c) p_{\mathrm{st}}(z)$ of the Fokker-Planck operator

$$
p\left(z, t \mid z_{0}, s\right)=p_{\mathrm{st}}(z) \sum_{n=0}^{\infty} \frac{n ! \Gamma(\mu)}{\Gamma(\mu+n)} L_{n}^{\mu-1}(z / c) L_{n}^{\mu-1}\left(z_{0} / c\right) \mathrm{e}^{-n \beta(t-s)}, \quad t \geqslant s,
$$

where $L_{n}^{\mu-1}(x)$ denote the generalized Laguerre polynomials, the paramaters $c$ and $\mu$ are defined by Eq. (4.25). Since the process $z(t)$ is Markovian, all finite-dimensional distributions are known provided that an initial distribution $p(z, 0)$ is given. We impose $p(z, 0)=p_{\mathrm{st}}(z)$. Then $p(z, t)=p_{\mathrm{st}}(z)$ and the statistical moments $\left\langle z^{n}(t)\right\rangle(n=1,2,3, \ldots)$ do not depend on time. In particular,

$$
\langle z(t)\rangle=\omega / \beta, \quad\left\langle z^{2}(t)\right\rangle-\langle z(t)\rangle^{2}=\omega \varepsilon / \beta^{2} .
$$

Using the spectral representation of the transition probability one obtains for the stationary correlation function:

$$
\begin{aligned}
\langle z(t) z(s)\rangle-\langle z(t)\rangle\langle z(s)\rangle & =\int_{0}^{\infty} \int_{0}^{\infty} z z_{0} p\left(z, t \mid z_{0}, s\right) p\left(z_{0}, s\right) \mathrm{d} z \mathrm{~d} z_{0}-(\omega / \beta)^{2} \\
& =\left(\omega \varepsilon / \beta^{2}\right) \mathrm{e}^{-\beta(t-s)}, \quad t>s .
\end{aligned}
$$

Accordingly, the process $z(t)$ is exponentially correlated with the correlation time $\tau_{c}=1 / \beta$. 


\section{References}

[1] J.R. Manning, Diffusion Kinetics for Atoms in Solids, Van Nostrand, Princeton, NJ, 1968.

[2] J. Philibert, Atom Movements, Diffusion and Mass transport in Solids, Les Editions de Physique, Cedex, 1991.

[3] A. Einstein, Investigations on the Theory of Brownian Movement, Dover, New York, 1956.

[4] J.A. McLennan, Introduction to Nonequilibrium Statistical Mechanics, Prentice-Hall, Englewood Cliffs, NJ, 1989.

[5] P. Meakin, in: C. Domb, J.L. Lebowitz (Eds.), Phase Transitions and Critical Phenomena, Vol. 12, Academic Press, London, 1998, p. 335.

[6] W. Hess, H.L. Frish, R. Klein, Z. Phys. B 64 (1986) 65.

[7] P. Meakin, J. Chem. Phys. 83 (1985) 3645.

[8] P. Wiltzius, Phys. Rev. Lett. 58 (1987) 710.

[9] R. Rozenfeld, J. Łuczka, P. Talkner, Phys. Lett. A 249 (1998) 409.

[10] P. Günberg, R. Schreiber, Y. Pang, M.B. Brodsky, H. Sowers, Phys. Rev. Lett. 57 (1986) 2442.

[11] R. van Helmholt, J. Wecker, B. Holzapfel, L. Schultz, K. Samwer, Phys. Rev. Lett. 71 (1993) 2331.

[12] J.-P. Bouchaud, A. Georges, Phys. Rep. 195 (1990) 12.

[13] P. Hänggi, Z. Phys. B 36 (1980) 271.

[14] P. Hänggi, Z. Phys. B 31 (1978) 407.

[15] A. Brissaud, U. Frisch, J. Math. Phys. 15 (1974) 524.

[16] H. Hochstadt, Integral Equations, Wiley, New York, 1973.

[17] E. Wong, Proc. Symp. Appl. Math. 16 (1964) 264.

[18] R.L. Stratonovich, Topics in the Theory of Random Noise, Vol. I, Gordon and Breach, New York, 1963. 